\title{
Doorway states in nuclear reactions as a manifestation of the "super-radiant" mechanism
}

\author{
N. Auerbach ${ }^{1}$ and V. Zelevinsky ${ }^{2}$ \\ ${ }^{1}$ School of Physics and Astronomy, Tel Aviv University, Tel Aviv 69978, Israel \\ ${ }^{2}$ National Superconducting Cyclotron Laboratory and Department of Physics and \\ Astronomy, Michigan State University, East Lansing, Michigan 48824-1321, USA
}

\begin{abstract}
A mechanism is considered for generating doorway states and intermediate structure in low-energy nuclear reactions as a result of collectivization of widths of unstable intrinsic states coupled to common decay channels. At the limit of strong continuum coupling, the segregation of broad ("super-radiating") and narrow ("trapped") states occurs revealing the separation of direct and compound processes. We discuss the conditions for the appearance of intermediate structure in this process and doorways related to certain decay channels.
\end{abstract}




\section{Introduction}

Apart from the two extremes of nuclear many-body dynamics, very narrow compound resonances and broad single-particle or giant resonances, nuclear cross sections exhibit other structures in between. The intermediate structures are related to more organized dynamics of nuclei as compared to the chaotic motion at the compound stage. There is a large variety of possible motion that gives rise to a diversity of intermediate structures.

The intermediate structure resonances in the nuclear cross sections have usually widths that are satisfying the following inequality:

$$
\Gamma_{q} \ll \Gamma_{\text {in }}<\Gamma_{\text {s.p. }} .
$$

Here $\Gamma_{q}$ denotes a width of a typical compound state, $\Gamma_{\text {s.p. }}$ that of a singleparticle resonance, and $\Gamma_{\text {in }}$ of the intermediate structure resonances.

Intermediate structure was often studied in the past using various approaches $[1,2,3,4,5]$, recently with the aid of the ideas of quantum chaos and wavelet analysis $[6,7,8]$. In the present work we apply the super-radiant (SR) approach $[9,10]$ to show how the intermediate structure emerges naturally in the theory. The SR approach has been used in many different fields. For example, it was applied to problems in chemistry [11, 12], atomic physics [13], condensed matter physics [14,10], intermediate energy nuclear physics [15, 16, 17], and most widely in the theory of nuclear reactions $[9,18,19,10]$, in particular to describe the chaotic dynamics of the compound nucleus and phenomena on the borderline of discrete and continuum spectra in loosely bound nuclei $[20,21]$. In the next section we will develop the SR formalism for some simple situations in order to arrive in a heuristic manner at the concept of intermediate structure.

\section{The SR mechanism and nuclear dynamics}

\subsection{The effective Hamiltonian}

Following the standard projection formalism [22], let us divide the Hilbert space of nuclear states into two parts, the $\{Q\}$-subspace involving very complicated many-body states $|q\rangle$, and the subspace $\{P\}$ of open channels $|c\rangle$. We use the notations $\mathcal{Q}$ and $\mathcal{P}$ for the corresponding projection operators onto the above subspaces. The total wave function of the system,

$$
|\Psi\rangle=\mathcal{Q}|\Psi\rangle+\mathcal{P}|\Psi\rangle,
$$

satisfies the stationary Schrödinger equation

$$
H|\Psi\rangle=E|\Psi\rangle,
$$

that can be decomposed into a set of coupled equations,

$$
\left(E-H_{Q Q}\right) \mathcal{Q}|\Psi\rangle=H_{Q P} \mathcal{P}|\Psi\rangle,
$$


and

$$
\left(E-H_{P P}\right) \mathcal{P}|\Psi\rangle=H_{P Q} \mathcal{Q}|\Psi\rangle,
$$

where we use the notations $H_{P Q}=\mathcal{P} H \mathcal{Q}$ and so on. Eliminating the part $\mathcal{P}|\Psi\rangle$, we come to the equation in the $Q$-space,

$$
\left(E-\mathcal{H}_{Q}\right) \mathcal{Q}|\Psi\rangle=0,
$$

with the effective Hamiltonian

$$
\mathcal{H}_{Q}=H_{Q Q}+H_{Q P} \frac{1}{E^{(+)}-H_{P P}} H_{P Q} .
$$

Here $E^{(+)} \equiv E+i 0$ contains the infinitesimal imaginary term $+i 0$ ensuring right asymptotic conditions for the continuum wave functions.

The second term of the effective Hamiltonian (7) contains a real and imaginary part of the propagator

$$
G^{(+)}(E)=\frac{1}{E^{(+)}-H_{P P}}
$$

emerging from the principal value (off-shell contributions) and the delta-function $\delta\left(E-H_{P P}\right)$ (on-shell contributions from channels $c$ open at energy $E$ ), respectively. The imaginary part, $-(i / 2) W$, of the effective Hamiltonian is given by

$$
W=2 \pi \sum_{c ; \text { open }} H_{Q P}|c\rangle\langle c| H_{P Q} .
$$

Thus, the effective Hamiltonian (7) in $Q$-space is non-Hermitian,

$$
\mathcal{H}_{Q}=\bar{H}-\frac{i}{2} W,
$$

where the total Hermitian part $\bar{H} \equiv \bar{H}_{Q Q}$ is a symmetric real matrix that includes, apart from the original Hamiltonian of $Q$-space, $H_{Q Q}$, the principal value contribution of the $Q P$-coupling and in realistic applications is given by the usual shell model, while the second part of (10) is anti-Hermitian.

The observable cross section of the reaction $a \rightarrow b$ is determined by the square of the scattering amplitude

$$
T^{b a}(E)=\sum_{q q^{\prime}}\left\langle a\left|H_{P Q}\right| q\right\rangle\left(\frac{1}{E^{(+)}-\mathcal{H}_{Q}}\right)_{q q^{\prime}}\left\langle q^{\prime}\left|H_{Q P}\right| b\right\rangle .
$$

Here the full propagator

$$
\mathcal{G}(E)=\frac{1}{E^{(+)}-\mathcal{H}_{Q}}
$$

includes all interactions between the subspaces that guarantees the unitarity of the scattering matrix, $S=1-i T$. The eigenvalues of $\mathcal{H}, \mathcal{E}=E-(i / 2) \Gamma$, are complex poles of the scattering matrix corresponding to the resonances in the cross sections. Here, however, one has to have in mind that the resulting picture in general can be complicated due to the interference of the resonances and dependence of the effective Hamiltonian on running energy $E$. 


\subsection{Single channel case}

To demonstrate in a simple way the effect of the anti-Hermitian term let us assume that only one channel is open. Then the matrix $W$, eq. (9), has a completely separable form,

$$
\left\langle q|W| q^{\prime}\right\rangle=2 \pi A_{q}^{c} A_{q^{\prime}}^{c *},
$$

where the amplitudes of the continuum coupling are

$$
A_{q}^{c}=\left\langle q\left|H_{Q P}\right| c\right\rangle .
$$

The rank of the factorized matrix $W$, eq. (13), equals 1 , so that all eigenvalues of $W$ are zero, except one that has the value equal to the trace $\Gamma_{0}$ of this matrix,

$$
\Gamma_{0}=\sum_{q}\langle q|W| q\rangle=2 \pi \sum_{q}\left|A_{q}^{c}\right|^{2} .
$$

Let us assume that the Hermitian part $\bar{H}$ of the effective Hamiltonian (10) is diagonalized, its eigenstates are $|g\rangle$ and the eigenvalues $E_{g}$ are degenerate. Making the additional diagonalization of $W$, we single out one superposition of the states $|g\rangle$, namely the decaying state with the width (15). while the orthogonal states are still degenerate and stable corresponding to the zero eigenvalues of $W$. The special unstable superposition of the eigenstates is often referred to as the super-radiant (SR), in analogy to the Dicke coherent state $[23,10]$ of a set of two-level atoms coupled through the common radiation field. Here the coherence is generated by the common decay channel. The stable states are trapped and decoupled from the continuum.

This result was obtained under strict assumptions. However, it is quite robust. In the eigenbasis $|g\rangle$ of the real part, the anti-Hermitian part is still factorized, the amplitudes $A_{q}^{c}$ are transformed to new values

$$
B_{g}^{c}=\left\langle g\left|H_{Q P}\right| c\right\rangle
$$

but the trace (15) is invariant. If the eigenstates $|g\rangle$ are not degenerate but the typical spacings $D$ in their spectrum are small, $D \ll\left\langle\gamma_{g}\right\rangle$, compared to the typical values of the unperturbed widths

$$
\gamma_{g}^{c}=2 \pi\left|B_{g}^{c}\right|^{2},
$$

the qualitative picture is essentially the same [24]. Among the eigenstates of the total non-Hermitian Hamiltonian, there is still one broad state and the rest of the states are very narrow. In fact, one can start with the diagonalization of $W$ [24]. In this doorway basis one state concentrates the whole width while the diagonalization of $\bar{H}$ in the orthogonal subspace defines the trapped states. The remaining Hermitian coupling transfers small widths to the trapped states. 


\subsection{General case}

The phenomenon of super-radiance survives in a general situation of $N$ intrinsic states and $N_{c}$ open channels provided $N_{c} \ll N$, if the mean level spacing $D$ of internal states and their characteristic decay widths $\gamma^{c}$ satisfy the same condition as earlier,

$$
\kappa^{c}=\frac{\gamma^{c}}{D}>1
$$

In the opposite limit, $\kappa^{c} \ll 1$, the anti-Hermitian part $W$ is a weak perturbation that provides small widths to intrinsic states $|g\rangle$ converting them into isolated narrow resonances. In this limit the quantities (17) are partial widths for a certain decay channel, and the total widths of resonances are given by

$$
\Gamma_{g}=\sum_{c} \gamma_{g}^{c}
$$

As the parameters $\kappa^{c}$ increase and reach the order of one, the influence of corresponding channels ceases to be a weak perturbation and the term $W$ starts to dominate the dynamics. The resonances go over to the overlapping regime and their interaction through the common continuum channels leads to the restructuring of the complex energy spectrum, similarly to the formation of the Dicke coherent state. The picture in this limit simplifies with the primary diagonalization of $W$. Since the rank of this factorized matrix is equal to $N_{c}$, it has only $N_{c}$ non-zero positive eigenvalues $w_{s}, s=1, \ldots, N_{c}$. As it is easy to derive from eq. (13), these nontrivial eigenvalues are at the same time the eigenvalues of the Hermitian $N_{c} \times N_{c}$ matrix in the channel space,

$$
X^{b a}=\sum_{q} A_{q}^{b *} A_{q}^{a} .
$$

The intrinsic space $\{Q\}$ is now divided into the $S R$ subspace $|s\rangle$ of dimension $N_{c}$ and the subspace of trapped states $|t\rangle$ of dimension $N-N_{c}$ that can couple to the continuum only through the Hermitian interaction $H_{s t}$ with the states $|s\rangle$ of the first class. In the regime of strong continuum coupling, the interaction between the two blocks is weak [24]. A simple perturbation theory gives small widths to the trapped states,

$$
\Gamma_{t}=\sum_{s=1}^{N_{c}} \frac{w_{s}\left|H_{s t}\right|^{2}}{\left(\Delta \epsilon_{s t}\right)^{2}+w_{s}^{2} / 4} .
$$

For example, returning again to the one-channel case $(s=1, t=2, \ldots, N)$ when the only non-zero eigenvalue of $W$ equals $w_{1}=\Gamma_{0}$, eq. (15), and the eigenvalues of $\bar{H}$ in the trapped block are $\epsilon_{t}$, we have

$$
\mathcal{H}_{11}=\epsilon_{1}-\frac{i}{2} \Gamma_{0},
$$


and the characteristic equation for complex energies $\mathcal{E}$ takes the form

$$
\mathcal{E}-\epsilon_{1}+\frac{i}{2} \Gamma_{0}-\sum_{t} \frac{\left|H_{1 t}\right|^{2}}{\mathcal{E}-\epsilon_{t}}=0 .
$$

In the limit (18), eq. (23) gives for the imaginary part of narrow states

$$
\Gamma_{t}=\Gamma_{0} \frac{\left|H_{1 t}\right|^{2}}{\left(\epsilon_{1}-\epsilon_{t}\right)^{2}+\Gamma_{0}^{2} / 4},
$$

in agreement with (21).

The segregation of the two classes of states can be interpreted as physical separation of the time scales corresponding to direct and compound processes. This separation of widths was noticed [25] in numerical calculations and explained in terms of the effective Hamiltonian in [9]. The segregation picture in the complex energy plane becomes spectacular in the many-channel case [19]. The representing set of resonances in the complex energy plane at $\left\langle\kappa^{c}\right\rangle \sim 1$ undergoes a sharp phase transition from the uniform cloud around the average small width to two well separated clusters, $\left(N-N_{c}\right)$ trapped states near the real energy axis and $N_{c}$ broad SR states.

\section{Doorways}

In order to see the emergence of intermediate structure we consider a frequent situation when only a subset of intrinsic states $\{Q\}$ connects directly to the $\{P\}$ space of channels. The rest of states in $\{Q\}$ will connect to $\{P\}$ only when they obtain admixtures of these selected states of the first type. The special states directly coupled to continuum are the doorways, $|d\rangle$. They form the doorway subspace $\{D\}$ within $\{Q\}$, and the corresponding projection operator will be denoted here as $\mathcal{D}$. The remaining states in $\{Q\}$ will be denoted as $|\tilde{q}\rangle$.

The decomposition of the full Hamiltonian now reads

$$
\begin{aligned}
H= & \left(H_{\tilde{Q} \tilde{Q}}+H_{D D}+H_{\tilde{Q} D}+H_{D \tilde{Q}}\right) \\
& +\left(H_{P P}+H_{D P}+H_{P D}\right) .
\end{aligned}
$$

Diagonalizing the operator in the upper line of (25) would give back the states $|g\rangle$ with the components of $|d\rangle$ mixed with $|\tilde{q}\rangle$ states. The two last items in the second line of (25) couple the states $|d\rangle$, and therefore all states $|g\rangle$, to the open channels $|c\rangle$.

\subsection{A single doorway}

We start with the case when there is only one important doorway state $|d\rangle$. The matrix elements of the effective operator $W$ in the intrinsic space are now given 
by

$$
\left\langle q|W| q^{\prime}\right\rangle=2 \pi \sum_{c=1}^{N_{c}}\left\langle q\left|H_{D P}\right| c\right\rangle\left\langle c\left|H_{P D}\right| q^{\prime}\right\rangle .
$$

Under the doorway assumption,

$$
\left\langle q\left|H_{D P}\right| c\right\rangle=\langle q \mid d\rangle\left\langle d\left|H_{D P}\right| c\right\rangle,
$$

where $\langle q \mid d\rangle$ is the admixture of the doorway to the state $|q\rangle$. Whence, eq. (26) reduces to

$$
\left\langle q|W| q^{\prime}\right\rangle=2 \pi\langle q \mid d\rangle\left\langle d \mid q^{\prime}\right\rangle \sum_{c}\left|\left\langle d\left|H_{D P}\right| c\right\rangle\right|^{2} .
$$

The matrix elements (2) are again separable, this time irrespective of the number $N_{c}$ of open channels. The doorway state serves as a single filter for coupling of the $\{Q\}$ space to the open channel space.

If the quantitative conditions for a super-radiant mechanism to work are satisfied (see the discussion below) we again find one broad state with a width

$$
\Gamma_{s}=2 \pi \sum_{q}|\langle q \mid d\rangle|^{2} \sum_{c}\left|\left\langle d\left|H_{D P}\right| c\right\rangle\right|^{2}=2 \pi \sum_{c}\left|\left\langle d\left|H_{D P}\right| c\right\rangle\right|^{2} .
$$

Naturally, the width $\Gamma_{s}$ is nothing but the total decay width, $\Gamma_{d}^{\uparrow}$, of the doorway into all open channels. The picture is essentially the same as in the case of one channel although the mechanism is different. The rest of the states in $\{Q\}$ remain stable in the limiting degenerate situation and have small widths if the degeneracy is not perfect.

\subsection{When is this picture valid?}

As we discussed, the criterion of validity is that the average spacing between the levels in $\{Q\}$ is smaller than the decay width of such a state "before" the SR mechanism was set at work. In relation to that, we can consider the spreading width, $\Gamma_{d}^{\downarrow}$, of the doorway state for the fragmentation into compound states $|\tilde{q}\rangle$. If $N_{q}$ is the number of compound states in the interval covered by the spreading width, their average energy spacing is

$$
\bar{D}_{q} \approx \frac{\Gamma_{d}^{\downarrow}}{N_{q}}
$$

Before the SR mechanism was turned on, the average decay width of a typical $|q\rangle$ state was

$$
\Gamma_{q}^{\uparrow}=2 \pi \sum_{c}\left|\left\langle q\left|H_{Q P}\right| c\right\rangle\right|^{2}
$$

that can be estimated as

$$
\Gamma_{q}^{\uparrow}=\frac{\Gamma_{s}}{N_{q}} .
$$


Therefore,

$$
\frac{\Gamma_{q}^{\uparrow}}{\bar{D}_{q}} \approx \frac{\Gamma_{s}}{\Gamma_{d}^{\downarrow}} \approx \frac{\Gamma_{d}^{\uparrow}}{\Gamma_{d}^{\downarrow}}
$$

We conclude that the requirement for the SR doorway mechanism to be valid can be formulated as

$$
\frac{\Gamma_{d}^{\uparrow}}{\Gamma_{d}^{\downarrow}}>1
$$

This condition is often satisfied, compare with the discussion of a "broad pole" in Refs. [26, 27].

\subsection{Total width of a doorway state}

Now we can show that the total observed width of a doorway state is in fact a simple sum of the continuum width $\Gamma_{d}^{\uparrow}$, eq. (29), and the spreading width $\Gamma_{d}^{\downarrow}$ used for estimates in the previous subsection.

The remaining states $|\tilde{q}\rangle$ of the $\{Q\}$ subspace play the role of the background. We can diagonalize this subset of $\{Q\}$ and get the states $|\nu\rangle$ as the eigenvectors of $H_{\tilde{Q} \tilde{Q}}$ and their unperturbed energies $E_{\nu}$. Let the matrix elements of the coupling of the states $|\nu\rangle$ with the doorway state be

$$
\left\langle d\left|H_{D \tilde{Q}}\right| \nu\right\rangle \equiv h_{\nu} .
$$

Then the characteristic equation for the complex energies in the $\{Q\}$ space is analogous to (23),

$$
\mathcal{E}-E_{d}+\frac{i}{2} \Gamma_{d}^{\uparrow}-\sum_{\nu} \frac{\left|h_{\nu}\right|^{2}}{\mathcal{E}-E_{\nu}}=0
$$

where $E_{d}$ is an unperturbed position of the doorway state given by the matrix element $\left\langle d\left|H_{D D}\right| d\right\rangle$.

Under the condition of validity (34), the sum in eq. (36) can be considered in a standard way [28]. Substituting this sum by an integral with a mean level density $\rho=1 / \bar{D}_{q}$, we acquire the real shift $\Delta$ of the doorway state and its effective spreading width given by the "golden rule",

$$
\Gamma_{d}^{\downarrow}=2 \pi \frac{|\bar{h}|^{2}}{\bar{D}_{q}} .
$$

The standard expression (37) is valid as long as the spreading width (37) does not exceed the energy interval $\Delta E$ within which the level density $\rho$ and the coupling matrix elements $h_{\nu}$ can be substituted by their average values [?, 30]. As a result, the doorway state is observed in the experiment that does not resolve the background structure of a resonance,

$$
\mathcal{E}_{d}=E_{d}+\Delta-\frac{i}{2}\left(\Gamma_{d}^{\uparrow}+\Gamma_{d}^{\downarrow}\right) .
$$


Here $\Delta$ and $\Gamma_{d}^{\downarrow}$ can be smooth function of energy taken in eq. (38) at a doorway centroid. We can mention also that if the background states have their own small continuum widths $\gamma$ (for example, related to slow evaporation processes), one needs to substitute in eq. (38) $\Gamma_{d}^{\uparrow}$ by $\Gamma_{d}^{\uparrow}-\gamma$ since the fragmentation of the doorway state depends on its real coupling to the background rather than on a total flow outside through the background.

\section{Examples}

\subsection{Isobaric analog state (IAS)}

The isobaric analog state (IAS), $|A\rangle$, is defined [31] as a result of action by the isospin lowering operator onto a parent state $|\pi\rangle$ of a certain isospin $T$,

$$
|A\rangle=\text { const } \cdot T_{-}|\pi\rangle \text {. }
$$

In a compound nucleus, the IAS is surrounded by many compound states $|q\rangle$ of lower isospin $T_{<}=T-1$. The Coulomb interaction violates the isospin purity fragmenting the strength of the IAS over many states $|q\rangle$ that results in the spreading width $\Gamma_{A}^{\downarrow}$ of the IAS. If located above thresholds, the IAS can also decay into several continuum channels that gives rise to the decay width $\Gamma_{A}^{\uparrow}$. In heavy nuclei, usually

$$
\Gamma_{A}^{\uparrow}>\Gamma_{A}^{\downarrow}
$$

For example, in the lead region, $\Gamma_{A}^{\uparrow} / \Gamma_{A}^{\downarrow} \approx 2[31,32]$.

The SR mechanism is therefore relevant to this case providing a straightforward explanation why the IAS appears as a single resonance with the decay width given by that of $|A\rangle$,

$$
\Gamma_{I A S}^{\uparrow}=2 \pi\left|\left\langle A\left|H_{Q P}\right| P\right\rangle\right|^{2} .
$$

\subsection{Single-particle resonance}

Let us consider a single-particle state $\left|\phi_{\text {s.p. }}\right\rangle$ that belongs to the space $\{Q\}$ but has energy embedded in the continuum (above the decay threshold). Such a single-particle state couples directly to the continuum channels, often through a one-body mean field potential $V$. Due to the residual interaction, the singleparticle state will be spread over complicated compound states. At low energy above threshold, the main coupling of the compound states $|q\rangle$ to the continuum channels takes place via the single-particle component $\left\langle\phi_{\text {s.p. }} \mid q\right\rangle$. Thus, the state $\left|\phi_{\text {s.p. }}\right\rangle$ serves as a doorway and therefore our discussion of the single doorway case applies to this situation. 
This mechanism will produce a state with a large single-particle width given by

$$
\Gamma_{\text {s.p. }}=2 \pi \sum_{c}\left|\left\langle\phi_{\text {s.p. }}|V| c\right\rangle\right|^{2} .
$$

This width should be identified with the width of single-particle resonances known in the framework of the optical potential in Feshbach theory [22]. The narrow neutron resonances enveloped by a single-particle resonances represent the trapped states. In the modern versions of the continuum shell model based on the effective non-Hermitian Hamiltonian [21], this approach, in the combination with the spectroscopic amplitudes describing the spreading into complicated configurations, allows one to find the widths of individual resonances and the particle cross section as a function of energy. Here the threshold energy dependence of the width (42) cannot be ignored [24, 21].

\section{Several channels and intermediate structure}

Here we return to the situation when the $|q\rangle$ states are coupled to a number of open channels in the $\{P\}$ space, a typical situation in nuclear physics. In addition to the elastic channel, the compound states in many cases can decay to excited states in the daughter nuclei.

The one-body decay channels can be described either as simple hole states in the daughter nucleus and a particle in the continuum or collective particle-hole (p-h) excitations of the daughter nucleus with the nucleon in the continuum. As mentioned above, in such situations the number of open channels is small compared to to the number of compound states. This leads to the correlations in the matrix elements of $W$, and as a result we expect a number of resonances with the widths that are of intermediate size between the compound widths and the single-particle width.

If $|h\rangle$ is a hole excitation in the daughter nucleus, and $|\tilde{p}\rangle$ the state of a nucleon in the continuum, the channel states can be presented as $|c\rangle=|h ; \tilde{p}\rangle$. Then the $W$ matrix takes the form

$$
\left\langle q|W| q^{\prime}\right\rangle=\sum_{h, \tilde{p}}\langle q|V| h ; \tilde{p}\rangle\left\langle h ; \tilde{p}|V| q^{\prime}\right\rangle .
$$

Among the various configurations contained in the $\{Q\}$ space there are the ones which have the particle-hole structure,

$$
|d\rangle=|h ; p\rangle,
$$

with the bound single-particle state $|p\rangle$. These states are mixed into the rest of compound states with the amplitudes $\langle q \mid h ; p\rangle$. This mixing will be dominated by the coupling of these components to the above defined channels $|h ; \tilde{p}\rangle$. Then 
the dominant contribution to the matrix elements in eq. (43) will be

$$
\left\langle q|W| q^{\prime}\right\rangle=\sum_{h, p, \tilde{p}}\langle q \mid h ; p\rangle\langle h ; p|V| h ; \tilde{p}\rangle \cdot\langle h ; \tilde{p}|V| h ; p\rangle\left\langle h ; p \mid q^{\prime}\right\rangle .
$$

The sum here includes also the states with the same hole $|h\rangle$ but different particle states $|p\rangle$.

We should stress here the difference between this case and the one discussed earlier of a single doorway and many channels. In the latter case, the matrix $W$ was factorized as $W_{q q^{\prime}}=a_{q} a_{q^{\prime}}^{*}$. In the present case the $W$ matrix cannot be written in this simple form because each channel has its own doorway(s) $|d\rangle$, so that the form of the matrix $W$ is

$$
W_{q q^{\prime}}=\sum_{d=1}^{N_{c}} a_{q}^{d} a_{q^{\prime}}^{d *}
$$

If $N_{c} \ll N_{q}$, we have correlations in $W$. This scenario of several open channels of specific nature leads to a cross section that exhibits resonances with widths $\Gamma_{\text {int }}$ of intermediate size, $\Gamma_{q} \ll \Gamma_{\text {int }}<\Gamma_{\text {s.p. }}$. Such resonances were observed in nuclear reactions long ago $[33,34]$ and the cross sections were said to exhibit intermediate structure $[35,36]$.

Intermediate structure of a different type, even for a single channel case, can arise as a signature of the presence of intrinsic states of different degree of complexity. This is a time-independent description of the conventional multi-step dynamics. Let us assume that the doorway state $|d\rangle$ is coupled directly only with a subset $|\nu\rangle$ of intrinsic states. Those can be, for example, particle-hole states with a straight connection to the single-particle resonance. There exist more complicated intrinsic states $|\lambda\rangle$ coupled with the p-h states through the matrix elements $h_{\nu \lambda}$ but decoupled from the doorway. This leads to the appearance of a hierarchy of spreading with the fragmentation of the first generation states $|\nu\rangle$. The standard consideration shows that, for incoherent couplings $h_{\nu \lambda}$, the propagator $G(E)=1 /(E-H)$ has in the p-h space matrix elements

$$
G_{\nu \nu}(E)=\frac{1+\left|h_{\nu}\right|^{2} /\left\{\left(E-\epsilon_{\nu}\right)\left[E-E_{d}+(i / 2) \Gamma_{d}^{\downarrow}\right]\right\}}{E-\epsilon_{\nu}+(i / 2) \Gamma_{\nu}^{\downarrow}} .
$$

Here, along with the spreading width $\Gamma_{d}^{\downarrow}$ of the doorway state, eq. (37), the spreading width of $\mathrm{p}$-h states appears,

$$
\Gamma_{\nu}^{\downarrow}=2 \pi \frac{\left\langle\left|h_{\nu \lambda}\right|^{2}\right\rangle}{D_{\lambda}}
$$

where $D_{\lambda}$ is the mean level spacing of the states $|\lambda\rangle$. Due to the large density $1 / D_{\lambda}$, the widths (48) of different states $|\nu\rangle$ should, as a rule, overlap, so that for 
their extraction from data one needs to perform additional statistical analysis, for instance along the lines of Refs. [6, 7, 8].

In some situations, several doorway states $|d\rangle$ appear separated by energy intervals larger than their total widths. The intrinsic states $|q\rangle$ couple to these doorways locally giving rise to spreading of doorways. According to the doorway hypothesis, the $|q\rangle$ states couple to the continuum only via the admixture(s) of the local doorways. Then the matrix $W$ separates into several disconnected quadratic blocks of dimensions $n_{d} \times n_{d}$ along the diagonal, where $n_{d}$ is the number of states $|q\rangle$ contained within the spreading width of the doorway $|d\rangle$.

For each such a submatrix, eq. (46) is valid, and the problem is reduced to that of the single doorway. The resulting picture is a series of resonances separated in energy with decay widths equal to the decay widths of individual doorways $|d\rangle$.

\section{Giant resonances}

Giant resonances in nuclei (or atomic clusters) give another example of similar physics [5]. Usually, the giant resonances are discussed in terms of collectivization of p-h configurations with identical spin-parity quantum numbers. The coherent residual interactions form a correlated state that carries much of the transition strength of a corresponding multipole operator. The giant resonances are mostly located in the particle continuum decaying via particle emission to the ground and excited states in the daughter nucleus.

Since the p-h giant resonance $|G\rangle$ is surrounded usually by a dense spectrum of $2 \mathrm{p}-2 \mathrm{~h}$ and more complex configurations, the residual strong interaction will mix $|G\rangle$ with this background. Each of the resulting states denoted as $|b\rangle$ will contain the admixture $\langle G \mid b\rangle$ of the giant resonance. The mixed states $|b\rangle$ couple to the continuum. If we assume that the dominant coupling is that through the admixture of the giant state, then $|G\rangle$ serves as a doorway, and the matrix $W$ is given by

$$
\left\langle b|W| b^{\prime}\right\rangle=\langle b \mid G\rangle\left\langle G \mid b^{\prime}\right\rangle \cdot \sum_{c=1}^{N_{c}}\langle G|V| c\rangle\langle c|V| G\rangle .
$$

Again, the matrix $W$ for the background states is of rank one, and the SR will emerge with the decay width

$$
\Gamma_{G}^{\uparrow}=2 \pi \sum_{c}|\langle G|V| c\rangle|^{2} .
$$

This is valid under the assumption that the energy spread of the background states $|b\rangle$ is small compared to their decay width,

$$
\Gamma_{b}^{\uparrow}=2 \pi \sum_{c}|\langle c|V| b\rangle|^{2} .
$$


As before, this condition can be expressed as

$$
\frac{\Gamma_{G}^{\uparrow}}{\Gamma_{G}^{\downarrow}}>1
$$

the spreading width of the giant resonance is smaller than its total decay width. When this condition is not satisfied, so that the spacings between the $2 p-2 h$ states are larger than their decay widths $\Gamma_{b}^{\uparrow}$, the situation of a single decay peak for the giant resonance might not hold. Still one could expect some bunching of background states to group in energy and have spacings within the group smaller than their decay widths $\Gamma_{b}^{\uparrow}$. Each such group then can be treated separately using the SR mechanism and appear as a single peak in the decay (or excitation) curve. Then one will observe intermediate structure resonances in the GR energy domain.

\section{Double humped potentials}

As a function of a collective coordinate, double (or even triple) humped potentials are often encountered, especially in heavy nuclei. For example, such structure in the dependence of collective energy on the quadrupole deformation parameter $\beta_{2}$ corresponds to normal-deformed and superdeformed wells. The first potential is usually deeper, and the decay of intrinsic states $|q\rangle$ in this potential is hampered because they have to penetrate the double barrier when decaying to the continuum.

The states defined in the first well can couple to a few states $|d\rangle$ in the shallower second potential that couples to the continuum through a single outer barrier. These states $|d\rangle$ serve as doorways for the decay of states $|q\rangle$ in the first potential well. Similar to the previous examples, the matrix $W$ can be written in the form

$$
\left\langle q|W| q^{\prime}\right\rangle=\sum_{c d d^{\prime}}\langle q \mid d\rangle\langle d|V| c\rangle\left\langle c|V| d^{\prime}\right\rangle\left\langle d^{\prime} \mid q^{\prime}\right\rangle .
$$

If the number of doorways $|d\rangle$ is small compared to the number of states $|q\rangle$, one should expect, as a result of diagonalization of the above matrix, several broader states with the rest of the states being narrow.

An outstanding example are the fission isomers $[37,38,28]$ in several heavy nuclei, such as ${ }^{241} \mathrm{Pu}$. The fission cross section in the neutron induced reaction on ${ }^{240} \mathrm{Pu}$ shows the structures with larger widths spaced several hundred $\mathrm{eV}$ apart, whereas the average spacing between the compound resonances is of the order of $\mathrm{eV}$. This phenomenon is interpreted as a direct consequence of a double humped potential in the fission process of a compound nucleus. The energy spacings between the few excited (usually, collective - rotational or vibrational) states in the second shallower potential are larger than between the compound states in the deeper well. When the compound states in the deeper well are in 
the energy vicinity of the states in the second well, they couple forming mixed states which, in turn, couple to the fission channel via the admixture of the state from the second well.

In the formalism we apply in this work, the states in the second minimum are the doorways $|d\rangle$ for the decay of a compound state into the open channels, in this case the fission ones, $|f\rangle$. The matrix $W$ for the compound states $|q\rangle$ in the vicinity of the doorway $|d\rangle$ can be written as

$$
\left\langle q|W| q^{\prime}\right\rangle=\langle q \mid d\rangle\left\langle d \mid q^{\prime}\right\rangle \sum_{f}|\langle d|V| f\rangle|^{2} .
$$

After the diagonalization of this matrix, one will find a state with a considerably wider decay width than that of a typical compound state but of course smaller than a single-particle decay width. The situation repeats itself for each of the states in the second well. As a result, one will observe intermediate structure in the fission cross section.

For the gamma decay from the second (superdeformed) well to the normaldeformed states, see [39] and references therein, the role of a similar doorway can be played by a collective multi-phonon state from the first well that has a large vibrational amplitude and therefore a noticeable tail stretched into the second well.

\section{Conclusion}

We have considered few examples justifying a generic qualitative picture of formation of doorway states and intermediate structures in various nuclear processes. The traditional theory of intermediate structure $[1,2,3]$ considers usually statistical sequences in time of multi-step particle interactions with possible precompound (or preequilibrium) decay to the continuum. The present formulation emphasizes complementary aspects of underlying physics, namely those that follow from the strict quantum-mechanical description of complicated many-body dynamics generated by the mean field and residual interactions. The approach based on the effective non-Hermitian Hamiltonian rigorously predicts the existence of different scales in the energy dependence of observed cross sections. These scales are defined by the intrinsic dynamics that combines collective (coherent) and chaotic (incoherent) features. The new collectivity responsible for the emergence of the doorways and related intermediate structure appears, under certain physical conditions, due to the factorized nature of corresponding terms in the effective Hamiltonian. This separability is deeply related to the properties of unitarity of the scattering matrix. In this way we obtain a supplementary description of many-body dynamics.

Support from the National Science Foundation grant PHY-0555366 and the grant from the Binational Science Foundation USA-Israel is gratefully acknowledged. 


\section{References}

[1] J.J. Griffin, Phys. Rev. Lett. 17, 478 (1966).

[2] H. Feshbach, A. Kerman, and S. Koonin, Ann. Phys. (N.Y.) 125, 429 (1980).

[3] W.A. Friedman, M.S. Hussein, K.W. McVoy, and P.A. Mello, Phys. Rep. 77, 47 (1981).

[4] S. Galès, Ch. Stoyanov and A.I. Vdovin, Phys. Rep. 166, 125 (1988).

[5] P.F. Bortignon, A. Bracco and R.A. Broglia, Giant Resonances: Nuclear Structure at Finite Temperature (Harwood Academic, Amsterdam, 1998).

[6] G. Kilgus, G. Kühner, S. Müller, A. Richter, and W. Knüpfer, Z. Phys. A 326, 41 (1987).

[7] A. Shevchenko et al., Phys. Rev. Lett. 93, 122501 (2004).

[8] Y. Kalmykov et al., Phys. Rev. Lett. 96, 012502 (2006).

[9] V.V. Sokolov and V.G. Zelevinsky, Nucl. Phys. A504, 562 (1989).

[10] A. Volya and V. Zelevinsky, in Nuclei and Mesoscopic Physics, ed. V. Zelevinsky, AIP Conference Proceedings 777, 2005, p. 229.

[11] V.B. Pavlov-Verevkin, Phys. Lett. A 129, 168 (1988).

[12] F. Remacle, M. Munster, V.B. Pavlov-Verevkin, and M. Desouter-Lecomte, Phys. Lett. A 145, 265 (1990).

[13] V.V. Flambaum, A.A. Gribakina, and G.F. Gribakin, Phys. Rev. A 54, 2066 (1996).

[14] K. Pichugin, H. Schanz, and P. Šeba, Phys. Rev. E 64, 056227 (2001).

[15] N. Auerbach, Phys. Rev. C 50, 1606 (1994).

[16] N. Auerbach and V. Zelevinsky, Phys. Rev. C 65 (2002) 034601.

[17] N. Auerbach, V. Zelevinsky, and A. Volya, Phys. Lett. B 590, 45 (2004).

[18] I. Rotter, Rep. Prog. Phys. 54, 635 (1991).

[19] F.M. Izrailev, D. Saher, and V.V. Sokolov, Phys. Rev. E 49, 130 (1994).

[20] A. Volya and V. Zelevinsky, Phys. Rev. C 67, 54322 (2003).

[21] A. Volya and V. Zelevinsky, Phys. Rev. Lett. 94 (2005) 052501. 
[22] H. Feshbach, Ann. Phys. 5, 357 (1958); 19, 287 (1962).

[23] R.H. Dicke, Phys. Rev. 93, 99 (1954).

[24] V.V. Sokolov and V.G. Zelevinsky, Ann. Phys. (N.Y.) 216, 323 (1992).

[25] P. Kleinwächter and I. Rotter, Phys. Rev. C 32, 1742 (1985).

[26] P. von Brentano, Z. Phys. A 306, 63 (1982).

[27] V.V. Sokolov and V. Zelevinsky, Phys. Rev. C 56, 311 (1997).

[28] A. Bohr and B.R. Mottelson, Nuclear Structure, vol. 2 (Benjamin, New York, 1989).

[29] C.H. Lewenkopf and V. Zelevinsky, Nucl. Phys. A569, 183c (1994).

[30] N. Frazier, B.A. Brown and V. Zelevinsky, Phys. Rev. C 54, 1665 (1996).

[31] N. Auerbach, J. Hüfner, A.K. Kerman, and C.M. Shakin, Rev. Mod. Phys. 44, 48 (1972).

[32] J. Reiter and H. L. Harney, Z. Phys. A337, 121 (1990).

[33] C.H. Holbrow and H.H. Barschall, Nucl. Phys. 42, 264 (1963).

[34] R.M. Wood, R.R. Borchers and H.H. Barschall, Nucl. Phys. 71, 529 (1965).

[35] A.K. Kerman, L.S. Rodberg, and J.E. Young, Phys. Rev. Lett. 11, 422 (1963).

[36] R.A. Ferrell and W.M. MacDonald, Phys. Rev. Lett. 16, 187 (1966).

[37] S.M. Polikanov, Sov. Phys. Usp. 94, 46 (1968).

[38] H. Weigmann, Zs. Phys. 214, 7 (1968).

[39] A.J. Sargeant, M.S. Hussein, and A.N. Wilson, in Nuclei and Mesoscopic Physics, ed. V. Zelevinsky, AIP Conference Proceedings 777 (2005) p. 46. 